\title{
Vitamin A deficiency is associated with severe Mycoplasma pneumoniae pneumonia in children
}

\author{
Yan Xing ${ }^{1 \#}$, Kai Sheng ${ }^{2 \#}$, Xiumei Xiao ${ }^{3 \#}$, Jiawei Li $^{1}$, Hongling Wei ${ }^{1}$, Ling Liu ${ }^{1}$, Wei Zhou ${ }^{1}$, Xiaomei Tong ${ }^{1}$ \\ ${ }^{1}$ Department of Pediatrics, Peking University Third Hospital, Beijing 100191, China; ${ }^{2}$ Department of Emergency, Beijing Children's Hospital, \\ Capital Medical University, Beijing 100045, China; ${ }^{3}$ Department of Laboratory Medicine, Peking University Third Hospital, Beijing 100191 , China \\ Contributions: (I) Conception and design: Y Xing, W Zhou, X Tong; (II) Administrative support: Y Xing, W Zhou, X Tong; (III) Provision of study \\ materials or patients: Y Xing, K Sheng, J Li, H Wei, L Liu; (IV) Collection and assembly of data: Y Xing, K Sheng, L Liu, X Xiao; (V) Data analysis \\ and interpretation: Y Xing, K Sheng; (VI) Manuscript writing: All authors; (VII) Final approval of manuscript: All authors. \\ \#These authors contributed equally to this work. \\ Correspondence to: Wei Zhou, MD; Xiaomei Tong, MD. Department of Pediatrics, Peking University Third Hospital, No. 49, Hua Yuan Bei Lu, \\ Haidian District, Beijing 100191, China. Email: weiz6553@vip.sina.com; tongxm2007@126.com.
}

Background: Children with vitamin A, D, and E deficiency are susceptible to respiratory infections. However, the correlations between the levels with Mycoplasma pneumoniae pneumonia (MPP) and patient MPP occurrence is still unclear. This study aims to measure and compare the serum levels in severe (sMPP) and non-severe MPP (nsMPP) and to investigate the correlations between their levels and the occurrence of MPP.

Methods: A total of 122 children were enrolled, including 52 sMPP and 70 nsMPP aged 0-15 years old in 2015-2018. The serum levels of vitamins A, D, and E were measured and compared, and two-category logistic regression was used for correlation analysis of vitamins A, D, and E levels with nsMPP and sMPP.

Results: The age was older (7.12 vs. $4.01 \mathrm{y}, \mathrm{P}=0.002)$ in the sMPP samples than that in the nsMPP samples. Vitamin A deficiency was present in both the nsMPP and sMPP samples; its level was significantly lower $(0.15 \pm 0.06$ vs. $0.19 \pm 0.07, \mathrm{P}=0.0193)$ in the sMPP serum than that in the nsMPP serum. Vitamins $\mathrm{E}$ and $\mathrm{D}$ in the sMPP samples were significantly lower (vitamin $\mathrm{E} 7.43 \pm 1.55$ vs. $8.22 \pm 2.22, \mathrm{P}=0.0104$; vitamin $\mathrm{D} 23.08 \pm 11.0$ vs. $32.07 \pm 19.2, \mathrm{P}=0.0007$ ) than that in the nsMPP group; both sMPP and nsMPP did not show a deficiency of vitamins $\mathrm{E}$ and $\mathrm{D}$. Logistic regression analysis revealed that vitamin A deficiency was significantly (OR 0.001, 95\% CI: 0.001-0.334, P=0.009) associated with sMPP, and vitamin A supplementation could reduce the incidence of sMPP. In $\geq 6$ y sMPP, the incidence of vitamin A deficiency was $62.5 \%$, while $<6$ y, $85 \%$, showing a significant difference. Vitamin A level in $<6$ y sMPP was significantly lower than that in $\geq 6$ y sMPP.

Conclusions: Vitamin A deficiency is associated with sMPP and more likely present in the younger sMPP samples. Therefore, it is important to watch and supplement vitamin A in M. pneumoniae infection patients.

Keywords: Vitamin A; vitamin D; vitamin E; Mycoplasma pneumoniae pneumonia (MPP)

Submitted Dec 20, 2019. Accepted for publication Jan 23, 2020.

doi: $10.21037 /$ atm.2020.02.33

View this article at: http://dx.doi.org/10.21037/atm.2020.02.33

\section{Introduction}

Mycoplasma pneumoniae (MP) infection is one of the vital aspects of community-acquired pneumonia, occurs throughout the year. MP, a common pathogen in pediatric respiratory diseases, can be transmitted by droplets (1).
Children of all ages are susceptible to MP infection (2). In recent years, the number of children with MP pneumonia (MPP) has increased and they have a prolonged course of the disease. Some typical symptoms include fever, wheezing, difficulty in breathing, chest pain, and chills. MPP, 
particularly refractory or severe MPP (sMPP), often result in pleural effusion, atelectasis, and another organ damage (3). Therefore, how to effectively reduce MPP incidence and improve its treatment and management has become a serious challenge to pediatricians in the clinic.

Presently, the pathogenesis of an MP infection is not completely clear. MP and human tissues such as heart, lung, liver, brain, kidney, and smooth muscle have some common antigens (4). It has been proposed that while an MP infection occurs, MP adheres to airway epithelial cells through membrane $\mathrm{P}$ protein, then causes an immune response by producing autoantibodies, forming immune complexes and activating complement, which leads to the release of toxins and thus causing respiratory and other target organs damage (4).

Vitamins $\mathrm{A}, \mathrm{D}$, and $\mathrm{E}$ are very common nutrients but necessary for normal metabolisms in the human body. Lack of vitamins can lead to a high incidence of respiratory and digestive diseases in patients, particularly in children. Vitamins also have a significant impact on disease prognosis $(2,5)$. Some studies reported that children with vitamins A, D, and $\mathrm{E}$ deficiency, even at a subclinical deficiency level, were more susceptible to a variety of respiratory infections (5). However, the nutritional status of vitamins A, D and E, as well as the relationship between their levels and MPP incidence, remains unclear. This study investigated and compared the serum levels of vitamins $\mathrm{A}, \mathrm{D}$, and $\mathrm{E}$ in hospitalized sMPP children with that in non-severe MPP (nsMPP). Our findings show that vitamin A deficiency was significantly correlated with sMPP, and more likely occurred in the younger sMPP.

\section{Methods}

\section{Study population}

In this study, a total of 122 children aged $0-15$ years with nsMPP or sMPP who were hospitalized in the Pediatric Department of Peking University Third Hospital from December 2015 to March 2018 were enrolled (Beijing, China). This study was conducted following the Declaration of Helsinki, and the Ethics approved the protocol of the Committee of the Peking University Third Hospital. Written informed consent was obtained from all participants.

Diagnostic criteria for nsMPP is serum MP $\operatorname{IgM}$ antibody $>1: 160$, or a single MP-IgM antibody positive, and no clinical and laboratory evidence for other pathogen infections. Diagnostic criteria for sMPP: based on nsMPP, patient should have the following presentations: (I) obvious shortness of breath or tachycardia (less than 1-year-old: $\mathrm{RR} \geq 50$ beats/min, HR $\geq 150$ beats/min; $1-5$ years old: $\mathrm{RR} \geq 40$ beats/min, HR $\geq 140$ beats/min; over 5 years old: $\mathrm{RR} \geq 30$ beats/min, HR $\geq 120$ beats/min), with or without arterial blood pressure drop (contraction pressure $\leq 75 \mathrm{mmHg}$ ), three concave signs and cyanosis; (II) use of macrolides for more than 1 week is ineffective (continuous temperature $\geq 38.5^{\circ} \mathrm{C}$ or lung imaging is not improved or even progress), or continuous fever for more than 10 days; (III) chest image shows large lamella dense shadow, occupying a segment of lung or above a lung lobe; (IV) accompanied by lung complications such as pleural effusion, atelectasis and lung necrosis/lung abscess; (V) severe hypoxemia $\left(\mathrm{PaO}_{2}<60 \mathrm{mmHg}\right)$ or other serious damage (central nervous system infection, heart failure, myocarditis, gastrointestinal bleeding, obvious electrolyte/acid-base balance disorder, etc.).

Children with congenital heart disease, chronic diarrhea, hereditary metabolic diseases, kidney diseases, congenital immune function defects, and long-term use of immunosuppressive agents were excluded.

\section{The measurement process used to evaluate serum vitamins $A, D$, and $E$}

Peripheral venous blood samples of all subjects were collected under fasting on the next day of admission. HPLC was used for measurement of serum vitamins $\mathrm{A}$ and $\mathrm{E}$, and the chemiluminescence method was used for measurement of serum vitamin $\mathrm{D}$. Detection of vitamins $\mathrm{A}, \mathrm{E}$, and $\mathrm{D}$ were performed in the Inpatient Laboratory of Peking University Third Hospital (Beijing, China). Vitamin A deficiency was defined by $<0.2 \mathrm{mg} / \mathrm{L}$, subclinical vitamin A deficiency by $0.2-0.3 \mathrm{mg} / \mathrm{L}$, and normal vitamin A level by $0.3-0.7 \mathrm{mg} / \mathrm{L}$. Vitamin E deficiency was defined by $<5 \mathrm{mg} / \mathrm{L}$, and normal vitamin $\mathrm{E}$ level by $5-20 \mathrm{mg} / \mathrm{L}$. Vitamin D deficiency was defined by $<15 \mathrm{ng} / \mathrm{mL}$, vitamin $\mathrm{D}$ insufficiency by $15.1-20 \mathrm{ng} / \mathrm{mL}$, and normal vitamin D level by $20.1-100 \mathrm{ng} / \mathrm{mL}$.

\section{Statistical analysis}

Data processing was performed using SPSS 16.0 statistical software (IBM, Armonk, NY, USA). Count data were presented with the number and percentages. The comparison between the two groups was statistically analyzed by chi-square test method. The non-normal 
Table 1 The general comparison between nsMPP and sMPP

\begin{tabular}{lcccc}
\hline Group & $\mathrm{n}$ & Age $(\mathrm{y})$ & $\mathrm{F} / \mathrm{M}$ & $\mathrm{BMI}\left(\mathrm{kg} / \mathrm{m}^{2}\right)$ \\
\hline nsMPP & 70 & $4.01(2.94-7.18)$ & $38 / 32$ & $15.32(14.47-17.21)$ \\
sMPP & 52 & $7.12(4.00-9.00)^{\mathrm{a}}$ & $24 / 28^{\mathrm{b}}$ & $14.92(14.47-17.01)^{\mathrm{C}}$ \\
\hline
\end{tabular}

a $, Z=-3.081, P=0.002 ;{ }^{b}, \chi^{2}=0.789, P=0.374 ;{ }^{c}, \chi^{2}=-0.725, P=0.469$. nsMPP, non-severe $M$. pneumoniae pneumonia; sMPP, severe $M$. pneumoniae pneumonia; $\mathrm{F}$, female, $\mathrm{M}$, male; BMI, body mass index.

Table 2 The serum levels of vitamins A, D, and $\mathrm{E}$ in nsMPP and sMPP

\begin{tabular}{lccc}
\hline Group & Vitamin A (mg/L) & Vitamin D $(\mathrm{ng} / \mathrm{mL})$ & Vitamin E (mg/L) \\
\hline nsMPP & $0.19 \pm 0.07$ & $32.07 \pm 19.2$ & $8.22 \pm 2.22$ \\
sMPP & $0.15 \pm 0.06$ & $23.08 \pm 11.0$ & $7.43 \pm 1.55$ \\
P & 0.0193 & 0.0007 & 0.0104 \\
\hline
\end{tabular}

Data are shown as mean \pm SD. $n=70$ (nsMPP) and 52 (sMPP). nsMPP, non-severe $M$. pneumoniae pneumonia; sMPP, severe $M$. pneumoniae pneumonia.

distribution measurement data was represented by $M(\mathrm{Q} 1$, Q3), and the Z-value was obtained by statistical analysis using the Mann-Whitney U Test (Wilcoxon Rank Sum Test). The factors that may affect the subjects with sMPP were selected by two-category logistic regression analysis. The difference was statistically significant at $\mathrm{P}<0.05$.

\section{Results}

\section{General comparison of nsMPP and sMPP}

In this study, 70 of nsMPP with median age $4.01 \mathrm{y}$ (interquartile range, 2.94-7.18 y) were recruited, including 32 of boys and 38 of girls, and 52 of sMPP with median age 7.12 y (interquartile range, 4-9 y) including 28 of boys and 24 of girls (Table 1). There was no significant difference of gender $(\mathrm{P}=0.374)$ and $\mathrm{BMI}(\mathrm{P}=0.469)$ between nsMPP and sMPP. A comparison of age shows a significant difference between nsMPP and sMPP. The age was obviously $(\mathrm{P}=0.002)$ older in sMPP than that in MPP, suggesting that the older children may be more vulnerable to sMPP than the youngers.

How serum levels of vitamins $A, E$, and $D$ between nsMPP and $\mathrm{SMPP}$ were compared

The serum levels of vitamins A, E, and D in nsMPP and sMPP were measured and compared as described. Both nsMPP and sMPP presented vitamin A deficiency with the average values of less than $0.20 \mathrm{mg} / \mathrm{L}$. Moreover, the level of vitamin A was significantly $(\mathrm{P}=0.0193)$ lower in sMPP than that in nsMPP (Table 2, Figure 1A).

The levels of vitamins $\mathrm{E}$ and $\mathrm{D}$ were also significantly $(\mathrm{P}<0.05)$ lower in sMPP than that in nsMPP, whereas both nsMPP and sMPP did not show deficiency of vitamins $\mathrm{E}$ and $\mathrm{D}$ with the average values of greater than $5 \mathrm{mg} / \mathrm{L}$ and $15 \mathrm{ng} / \mathrm{mL}$, respectively (Table 2, Figure 1B,C).

How the logistic regression analysis of vitamins $A, D$, and E in sMPP was performed

Because of the statistical difference of age between nsMPP and sMPP, vitamins A, D, E, and age were separately screened by binomial logistic regression to name the potential factors that may affect sMPP incidence. Our analysis data show that vitamin A deficiency was associated with sMPP (OR 0.001, 95\% CI: 0.001-0.334, P=0.009) (Table 3).

\section{How the comparison of serum vitamin $A$ level in sMPP and nsMPP with different age was determined}

Based on age, sMPP was further classified into two groups: Group 1 ( $\geq 6$ y) and Group 2 ( $<6$ y). In Group 1, the occurrence rate of vitamin A deficiency was 20/32 (62.5\%), while in Group 2, 17/20 (85\%), showing a significant difference $\left(\chi^{2}=4.731, \mathrm{P}=0.030\right)$. Moreover, the level of vitamin A was significantly lower $(0.14 \pm 0.06$ vs. $0.18 \pm 0.08$, $\mathrm{P}=0.0208$ ) in Group 2 than that in Group 1 (Figure 2A). 

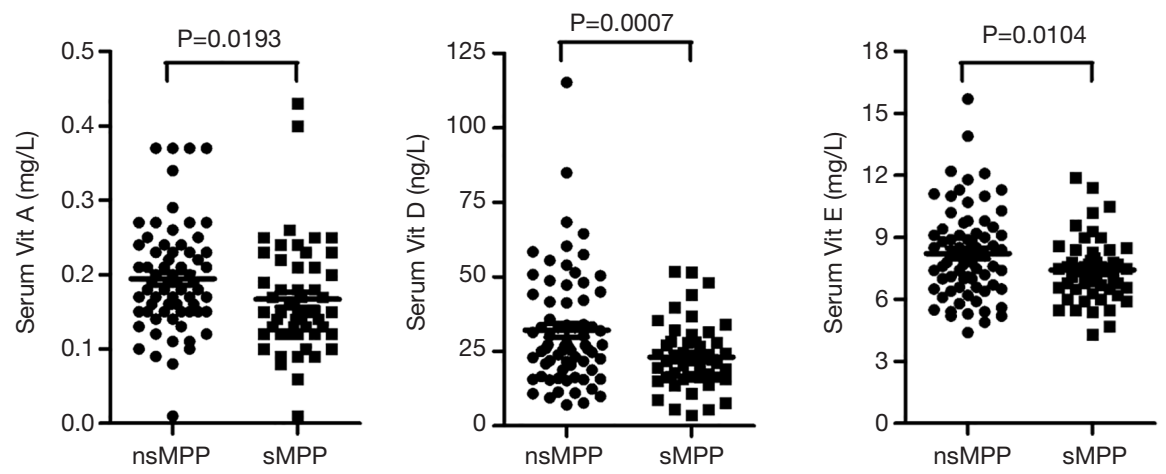

Figure 1 Comparison of the serum levels of vitamins A, D, and E between nsMPP and sMPP. The serum levels of vitamins A, D, and E were measured and compared between nsMPP and sMPP. Data are shown as mean \pm SD. n=70 (nsMPP) and 52 (sMPP). nsMPP, non-severe M. pneumoniae pneumonia; sMPP, severe M. pneumoniae pneumonia.

Table 3 Logistic regression analysis of vitamins A, D, and E in sMPP

\begin{tabular}{lcclll}
\hline Index & B & S.E. & Wald & OR $(95 \%$ Cl) & P \\
\hline Vitamin A & -7.874 & 2.996 & 6.907 & $0.001(0.001-0.334)$ & 0.009 \\
Vitamin E & -0.182 & 0.107 & 2.914 & $0.834(0.676-1.027)$ & 0.088 \\
Vitamin D & -0.182 & 0.019 & 2.817 & $0.969(0.934-1.005)$ & 0.093 \\
\hline
\end{tabular}

sMPP, severe M. pneumoniae pneumonia; B, the coefficient for the constant in the null model; S.E., standard error around the coefficient for the constant; Wald, the Wald chi-square test value; OR, odds ratio; $\mathrm{Cl}$, confidence interval.
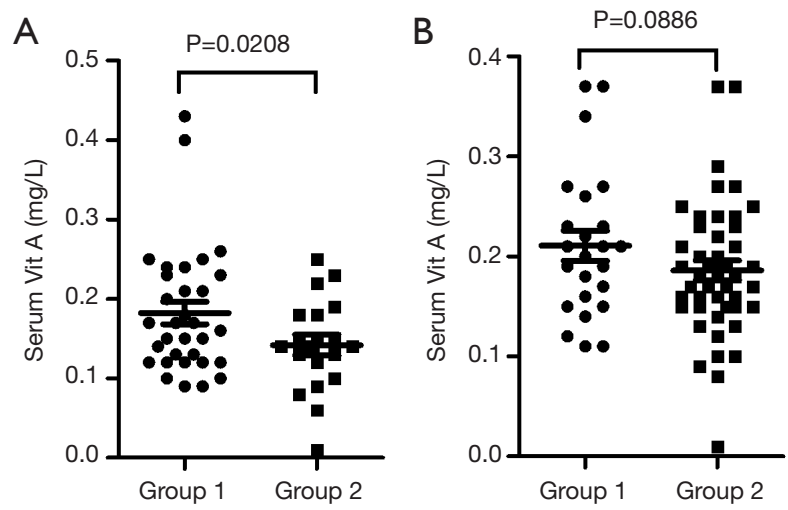

Figure 2 Comparison of the serum vitamin A levels in nsMPP and sMPP of different ages. (A) Patients with sMPP were divided into two groups: Group 1 ( $\geq 6 \mathrm{y}$ ) and Group 2 ( $<6$ y). The serum level of vitamin A was significantly lower in Group 2 than that in Group 1 . Data are shown as mean \pm SD. $n=32$ (Group 1) and 20 (Group 2); (B) patients with nsMPP were divided into two groups: Group 1 ( $\geq 6$ y) and Group 2 (<6 y). The serum level of vitamin A showed no difference between Group 1 and Group 2. Data are shown as mean \pm SD. $\mathrm{n}=24$ (Group 1 ) and 46 (Group 2). nsMPP, non-severe M. pneumoniae pneumonia; sMPP, severe $M$. pneumoniae pneumonia. 
Based on age, nsMPP was also classified into two groups: Group 1 ( $\geq 6$ y) and Group 2 ( $<6$ y). In Group 1, the occurrence rate of vitamin A deficiency was 12/24 (50\%), while in Group 2, 28/46 (60.9\%), showing no significant difference $\left(\chi^{2}=0.761, P=0.383\right)$. However, the level of vitamin $\mathrm{A}$ did not show significant difference $(0.21 \pm 0.074$ vs. $0.19 \pm 0.068, \mathrm{P}=0.0886$ ) between Group 1 and Group 2 (Figure 2B).

\section{Discussion}

In this current study, we analyzed the serum levels of vitamins $\mathrm{A}, \mathrm{D}$, and $\mathrm{E}$ in children with nsMPP and sMPP, and investigated their potential correlation with the incidence of nsMPP and sMPP. We found that both nsMPP and sMPP patients had a vitamin A deficiency. It has been well known that vitamin A, a fat-soluble micronutrient, is essential for the growth and differentiation of epithelial tissues.

Vitamin A plays an important pleiotropic role in supporting the normal mucosal barrier (6). It was found that vitamin A deficiency causes squamous metaplasia of the respiratory epithelium, impairment of airway mucosal barrier, delay of repair, and reduction of airway antioxidants, with the result of the increase of the risk of invasive pathogens (7). Children who presented vitamin A deficiency were more likely to get an acute respiratory tract infection and diarrhea (8). MP infection can increase the infiltration of inflammatory cells, the release of various cytokines such as IL-13 and IL-10, and hyperpermeability of airway epithelial cells (9). It was also reported that vitamin A differentially regulates cytokine such as IL-6, MCP-1, and IL-10 expressions in respiratory epithelial and macrophage cell lines infected with LPS or Sendai virus (10). In vitamin-A deficient rats, the number of mucosal dendritic cells and the production of IL-12 markedly increased; the mucosal expressions of Toll-like receptor 2 and MyD88 were up-regulated, and secretions of interferon- $\gamma$ and secretory IgA were decreased. Therefore, an infection aggravated the damage to the intestinal mucosa and lowered immunity in vitamin-A deficient rats (11). The functions of macrophages, neutrophils and natural killer cells were impaired, and the protective function of the respiratory mucosa was also decreased in the deficiency of vitamin A (12). Also, the extracellular matrix supplies enough strength and toughness support for tissue in gas exchange, and their morphological changes may be contributing factors to the development and progression of lung disease. Vitamin A deficiency can lead to changes in the extracellular matrix and basement membrane protein content and distribution. It was reported that the abundances of collagen I and IV were increased in the lungs of rats with vitamin A deficiency, leading to a double increase in the thickness of alveolar BM and deposition of ectopic type I collagen fibers (13-15).

The incidence and severity of infectious diseases are increased in vitamin A deficiency children $(1,2)$. Epidemiological surveys show that the incidence of vitamin A deficiency is significantly associated with age; the younger the age, the higher the incidence of vitamin A deficiency $(1,2)$. However, the correlation between vitamin A levels and nsMPP or sMPP occurrence is still unclear. The present study shows that the level of vitamin A was significantly lower in SMPP than that in nsMPP and that the older children were more vulnerable to sMPP than the youngers. There was an in vitro study showing that vitamin A deficiency is related to MP infections. The addition of vitamin A to MP-infected A549 cells can reduce the secretion of IFN- $\gamma$ and inflammatory factors (16). In our study, two binomial logistic regression analysis showed that vitamin A deficiency was associated with sMPP, and the supplementation of vitamin A may reduce the occurrence of sMPP in children. Several studies also showed that supplementation of vitamin A could reduce the incidence of respiratory infections and shorten the course of the disease (17-19). Moreover, we also found that vitamin A deficiency was more likely present in younger sMPP children $(<6$ y) compared to the $>6$ y sMPP. This finding suggests that supplementation of vitamin A is more important in younger children who have potential MP infection.

Besides, we found that the levels of vitamins $\mathrm{E}$ and D were lower in sMPP than that in nsMPP, while both nsMPP and sMPP did not show vitamin $\mathrm{E}$ and $\mathrm{D}$ deficiency. Vitamin E plays a critical role in antioxidant defense systems. Children who presented vitamin E deficiency were more likely to get an acute respiratory tract infection (15). In cultured fibroblasts infected by MP, oxidative damage was detected, presenting with an increase in intracellular levels of $\mathrm{H}_{2} \mathrm{O}_{2}$ and $\mathrm{O}_{2}^{-}$, which was limited by supplementation of vitamin E (20). One study from Ethiopia shows that preschool girls with low $25(\mathrm{OH}) \mathrm{D}$ levels were more vulnerable to pathogenic microbes such as influenza, respiratory viruses, and airway pathogenic bacteria than boys and that vitamin $\mathrm{D}$ deficiency was associated with the increased use of antimicrobials among preschool girls (21). The deficiency of vitamin $\mathrm{A}, \mathrm{D}$, and $\mathrm{E}$ is positively correlated with the disease activity of children with a 
recurrent respiratory infection, and interestingly, vitamins $\mathrm{E}$ and A were positively correlated with vitamin D (5). In our study, although we did not find a significant correlation between vitamins $\mathrm{E}$ and D levels with nsMPP and sMPP, vitamins $E$ and $D$ should be maintained at least at the normal levels since we found that they were significantly lower in sMPP than nsMPP.

\section{Conclusions}

Our data shows that vitamin A deficiency is associated with the onset of sMPP, particularly in younger children, and the addition of vitamin A can reduce the incidence of sMPP. It is important to check and supplement vitamin A in MP infection patients. The relationship between vitamins $\mathrm{E}$ and D levels and MPP or sMPP occurrence should be further investigated.

\section{Acknowledgments}

Funding: This project was funded by a research grant from Beijing Natural Science Foundation (No. 7163232 and No. S170003 to YX, No. S160004 to XT), the Young Scientists Fund of Peking University Third Hospital (No. BYSY2016016 to YX) and Medical and Health Science and Technology Development Research Center of National Health and Family Planning Commission (No. W2015EAE016 to YX).

\section{Footnote}

Conflicts of Interest: The authors have no conflicts of interest to declare.

Ethical Statement: The authors are accountable for all aspects of the work in ensuring that questions related to the accuracy or integrity of any part of the work are appropriately investigated and resolved. The informed consent was granted by all patients, and the Ethics approved these study protocols of the Committee of the Peking University Third Hospital (No. IRB00006761-2016010).

\section{References}

1. Lee YH, Seo H, Cha SI, et al. A case of pseudomembranous tracheitis caused by Mycoplasma pneumoniae in an immunocompetent patient. Ann Transl Med 2019;7:205.
2. Meyer Sauteur PM, Unger WW, Nadal D, et al. Infection with and Carriage of Mycoplasma pneumoniae in Children. Front Microbiol 2016;7:329.

3. Narita M. Pathogenesis of neurologic manifestations of Mycoplasma pneumoniae infection. Pediatr Neurol 2009;41:159-66.

4. Waites KB, Talkington DF. Mycoplasma pneumoniae and its role as a human pathogen. Clin Microbiol Rev 2004;17:697-728.

5. Zhang J, Sun RR, Yan ZX, et al. Correlation of serum vitamin $\mathrm{A}, \mathrm{D}$, and $\mathrm{E}$ with recurrent respiratory infection in children. Eur Rev Med Pharmacol Sci 2019;23:8133-8.

6. Sirisinha $\mathrm{S}$. The pleiotropic role of vitamin $\mathrm{A}$ in regulating mucosal immunity. Asian Pac J Allergy Immunol 2015;33:71-89.

7. Cassani B, Villablanca EJ, De Calisto J, et al. Vitamin A and immune regulation: role of retinoic acid in gutassociated dendritic cell education, immune protection and tolerance. Mol Aspects Med 2012;33:63-76.

8. Qi YJ, Niu QL, Zhu XL, et al. Relationship between deficiencies in vitamin $\mathrm{A}$ and $\mathrm{E}$ and occurrence of infectious diseases among children. Eur Rev Med Pharmacol Sci 2016;20:5009-12.

9. Thavagnanam S, Parker JC, McBrien ME, et al. Effects of IL-13 on mucociliary differentiation of pediatric asthmatic bronchial epithelial cells. Pediatr Res 2011;69:95-100.

10. Penkert RR, Jones BG, Häcker H, et al. Vitamin A differentially regulates cytokine expression in respiratory epithelial and macrophage cell lines. Cytokine 2017;91:1-5.

11. Yang $Y$, Yuan $Y$, Tao $Y$, et al. Effects of vitamin A deficiency on mucosal immunity and response to intestinal infection in rats. Nutrition 2011;27:227-32.

12. Timoneda J, Rodríguez-Fernández L, Zaragozá R, et al. Vitamin A Deficiency and the Lung. Nutrients 2018;10:pii:E1132.

13. Schwartz E, Zelig R, Parker A, et al. Vitamin A Supplementation for the Prevention of Bronchopulmonary Dysplasia in Preterm Infants: An Update. Nutr Clin Pract 2017;32:346-53.

14. Esteban-Pretel G, Marín MP, Renau-Piqueras J, et al. Vitamin A deficiency disturbs collagen IV and laminin composition and decreases matrix metalloproteinase concentrations in rat lung. Partial reversibility by retinoic acid. J Nutr Biochem 2013;24:137-45.

15. Esteban-Pretel G, Marín MP, Renau-Piqueras J, et al. Vitamin A deficiency alters rat lung alveolar basement membrane: reversibility by retinoic acid. J Nutr Biochem 2010;21:227-36. 
16. Wu X, Liu X, Tang J. The effect of vitamin A on secretion of IFN-gamma and IL-4 in A549 cells induced by Mycoplasma pneumoniae. J Huazhong Univ Sci Technolog Med Sci 2008;28:649-52.

17. Cameron C, Dallaire F, Vézina C, et al. Neonatal vitamin A deficiency and its impact on acute respiratory infections among preschool Inuit children. Can J Public Health 2008;99:102-6.

18. Hu N, Li QB, Zou SY. Effect of vitamin A as an adjuvant therapy for pneumonia in children: a Meta analysis. Zhongguo Dang Dai Er Ke Za Zhi 2018;20:146-53.

19. Imdad A, Mayo-Wilson E, Herzer K, et al. Vitamin A

Cite this article as: Xing Y, Sheng K, Xiao X, Li J, Wei H, Liu L, Zhou W, Tong X. Vitamin A deficiency is associated with severe Mycoplasma pneumoniae pneumonia in children. Ann Transl Med 2020;8(4):120. doi: 10.21037/atm.2020.02.33 supplementation for preventing morbidity and mortality in children from six months to five years of age. Cochrane Database Syst Rev 2017;3:CD008524.

20. Almagor M, Kahane I, Gilon C, et al. Protective effects of the glutathione redox cycle and vitamin $\mathrm{E}$ on cultured fibroblasts infected by Mycoplasma pneumoniae. Infect Immun 1986;52:240-4.

21. Bodin J, Mihret A, Holm-Hansen C, et al. Vitamin D Deficiency is Associated with Increased Use of Antimicrobials among Preschool Girls in Ethiopia. Nutrients 2019;11:pii: E575. 